\begin{tabular}{lll} 
C O L L O Q U I U M & M A T H E M A T I C U M \\
\hline VOL. LXVIII & 1995 & FASC. 2 \\
\hline
\end{tabular}

\title{
LINEAR COMBINATIONS OF CANTOR SETS
}

BY

J. E. NYMANN (EL PASO, TEXAS)

1. Introduction. At least three recent papers [7], [8], [9] have been concerned with the (previously known) fact that $C+C=[0,2]$ where $C$ is the Cantor ternary set. This paper will be concerned with investigating the topological structure of some sets of the form $\alpha_{1} E+\ldots+\alpha_{n} E$ where $\alpha_{1}, \ldots, \alpha_{n}$ are real numbers and $E$ is in a certain class of sets which includes $C$ and many sets homeomorphic to $C$.

Let $\sum a_{n}$ be a convergent series with $0<a_{n+1} \leq a_{n}$ for all $n$ and let

$$
E=\left\{\sum \varepsilon_{n} a_{n}: \varepsilon_{n}=0 \text { or } 1(n=1,2, \ldots)\right\}
$$

denote its set of subsums. Also let

$$
r_{n}=\sum_{k=n+1}^{\infty} a_{k}
$$

denote the $n$th "tail" of the series.

The following three facts (which will be needed later in this paper) were discovered in 1914 by Kakeya [4], and rediscovered by Hornich [3] in 1941 (see also the papers by Barone [1], Koshi and Lai [5] and Menon [6]):

A. $E$ is a perfect set.

B. $E$ is a finite union of closed intervals if and only if $a_{n} \leq r_{n}$ for $n$ sufficiently large. (Also, $E$ is an interval if and only if $a_{n} \leq r_{n}$ for all $n$.)

C. If $a_{n}>r_{n}$ for $n$ sufficiently large, then $E$ is homeomorphic to the Cantor set.

In a 1988 paper [2] the following theorem, which characterizes the topological structure of $E$, was proved.

THEOREM 1. If $E$ is the set of subsums of a positive term convergent series, then $E$ is one of the following:

(i) a finite union of closed intervals;

1991 Mathematics Subject Classification: Primary 11B05. 
(ii) homeomorphic to the Cantor set $C$;

(iii) homeomorphic to the set $T$ of subsums of $\sum b_{n}$ where $b_{2 n-1}=3 / 4^{n}$ and $b_{2 n}=2 / 4^{n}(n=1,2,3, \ldots)$.

Remark 1. In order to help visualize the set $T$ above, we give the following example of a set which is homeomorphic to $T$ (see [2]). Let $S_{n}$ denote the union of the $2^{n-1}$ open middle thirds which are removed from $[0,1]$ at the $n$th step in the construction of $C$. Then

$$
C=[0,1] \backslash \bigcup_{n=1}^{\infty} S_{n} .
$$

$T$ is homeomorphic to $C \cup \bigcup_{n=1}^{\infty} S_{2 n-1}$.

R e m ark 2. Statement B above gives necessary and sufficient conditions for $E$ to be a set of type (i) in Theorem 1. An interesting unsolved problem is to determine similar conditions for $E$ to be of type (ii).

2. Topological structure of $E+\ldots+E$. For any set $S$ of real numbers and $m$ any positive integer, we let

$$
\bigoplus_{m} S=\left\{s_{1}+\ldots+s_{m}: s_{i} \in S(i=1, \ldots, m)\right\}
$$

denote the algebraic sum of $S$ with itself $m$ times. Then using the notation of Section 1 for $a_{n}, r_{n}$ and $E$ we have

THEOREM 2. There is a positive integer $m$ for which $\bigoplus_{m} E$ is a finite union of intervals if and only if

$$
\lim \sup a_{n} / r_{n}<\infty \text {. }
$$

Moreover, the smallest positive integer $m$ for which $\bigoplus_{m} E$ is a finite union of intervals is the smallest integer $m$ such that $a_{n} / r_{n} \leq m$ for all but a finite number of integers $n$.

Proof. Let $m$ be a fixed positive integer. Construct a new sequence $\left\langle c_{n}\right\rangle$ such that

$$
c_{(q-1) m+1}=c_{(q-1) m+2}=\ldots=c_{q m}=a_{q}
$$

for $q=1,2, \ldots$ Note that $\sum c_{n}$ is a convergent series, $0<c_{n+1} \leq c_{n}$ for all $n$ and $\bigoplus_{m} E$ is the set of subsums of $\sum c_{n}$. Let

$$
\bar{r}_{n}=\sum_{k=n+1}^{\infty} c_{k}
$$

denote the $n$th tail of $\sum c_{n}$. Then by statement $\mathrm{B}$ above, $\bigoplus_{m} E$ is a finite union of intervals if and only if $c_{n} \leq \bar{r}_{n}$ for all but a finite number of integers $n$. This inequality is clearly true if $m \neq \equiv 0(\bmod n)$. Now if $n=m q$, then $c_{n}=a_{q}$ and $\bar{r}_{n}=m r_{q}$. Therefore $\bigoplus_{m} E$ is an interval if and only if 
$a_{q} \leq m r_{q}$ for all but a finite number of integers $q$. The theorem is now clear.

With slight modifications in the above proof we have the following special case of Theorem 2.

Corollary 1. There is a positive integer $m$ for which $\bigoplus_{m} E$ is an interval if and only if

$$
\lim \sup a_{n} / r_{n}<\infty .
$$

Moreover, the smallest integer $m$ for which $\bigoplus_{m} E$ is an interval is the smallest integer $m$ for which $a_{n} / r_{n} \leq m$ for all $n$.

Remark 3. The Cantor set $C$ is the set of subsums of $\sum a_{n}$ with $a_{n}=$ $2 / 3^{n}$. For this series $a_{n} / r_{n}=2$ for all $m$. Hence by Corollary $1, \bigoplus_{2} C=$ $C+C$ is an interval and the interval is clearly $[0,2]$.

EXAMPLE 1. We now give an example of a series $\sum a_{n}$ for which $\bigoplus_{m} E$ is not an interval for any $m$. Let $a_{n}=2^{-n^{2}}$. Then

$$
r_{n}=\sum_{k=n+1}^{\infty} 2^{-k^{2}}=\sum_{i=1}^{\infty} 2^{-(n+i)^{2}}<2^{-(n+1)^{2}} \sum_{i=1}^{\infty} 2^{-i+1}=2 \cdot 2^{-(n+1)^{2}} .
$$

Hence

$$
a_{n} / r_{n}>2^{-n^{2}} /\left(2 \cdot 2^{-(n+1)^{2}}\right)=2^{2 n} \rightarrow \infty
$$

as $n \rightarrow \infty$, and by Theorem $2, \bigoplus_{m} E$ is not an interval for any $m$.

Remark 4. Since $\bigoplus_{m} E$ is the set of the subsums of the series $\sum c_{n}$ constructed in the proof of Theorem 2, we see from Theorem 1 that $\bigoplus_{m} E$ is always a set of one of the three types given in Theorem 1 .

3. Topological structure of $a E+b E$. As before, $E$ will denote the set of subsums of $\sum a_{n}$ where $0<a_{n+1} \leq a_{n}$ for all $n$. Letting $s$ denote the sum of the series, the following facts are easy to check.

(1) $E=s-E$.

(2) $a E+b E=a E+(-b) E+b s$ for $a, b$ any real numbers.

(3) $a E+b E=a(E+(b / a) E)$ for $a, b$ any real numbers.

In this section we will be concerned with exploring the topological structure of $a E+b E$ for arbitrary real numbers $a$ and $b$. By (2) and (3) above, it is sufficient to study the topological structure of $E+x E$ for $0 \leq x \leq 1$.

TheOREM 3. Assume $a_{n+1} / a_{n}<x \leq 1$ for all $n$.

(i) $E+x E$ is an interval if and only if

$$
\left(a_{n}-r_{n}\right) / r_{n-1} \leq x \leq r_{n} /\left(a_{n}-r_{n}\right)
$$

for all $n$ for which $a_{n}>r_{n}$. 
(ii) $E+x E$ is homeomorphic to the Cantor set if

$$
r_{n} /\left(a_{n}-r_{n}\right)<x<\left(a_{n}-r_{n}\right) / r_{n-1}
$$

for $n$ sufficiently large.

Proof. Construct a new sequence $\left\langle c_{n}\right\rangle$ for which $c_{2 n-1}=a_{n}$ and $c_{2 n}=$ $x a_{n}$ for $n=1,2, \ldots$ and note that $c_{n+1} \leq c_{n}$ since $a_{n+1} / a_{n} \leq x \leq 1$. Also note that $E+x E$ is the set of subsums of $\sum c_{n}$. Let $\bar{r}_{n}=\sum_{k=n+1}^{\infty} c_{n}$ denote the $n$th tail of $\sum c_{n}$. Now by statement $\mathrm{B}, E+x E$ is an interval if and only if $c_{n} \leq \bar{r}_{n}$ for all $n$. It is easy to check that $\bar{r}_{2 n}=(1+x) r_{n}$ and $\bar{r}_{2 n-1}=x a_{n}+(1+x) r_{n}$. Hence $E+x E$ is an interval if and only if $x a_{n} \leq(1+x) r_{n}$ and $a_{n} \leq x a_{n}+(1+x) r_{n}$ for all $n$. The first part of the theorem follows immediately.

Similarly, by statement C, $E+x E$ is homeomorphic to the Cantor set if $c_{n}>\bar{r}_{n}$ for $n$ sufficiently large. Hence $E+x E$ is homeomorphic to the Cantor set if $x a_{n}>(1+x) r_{n}$ and $a_{n}>x a_{n}+(1+x) r_{n}$ for $n$ sufficiently large. Part (ii) is now clear.

In what follows we specialize to the case $a_{n}=a^{n}$ with $0<a<1$ and denote the set of subsums of $\sum a^{n}$ by $E_{a}$. By statements $\mathrm{B}$ and $\mathrm{C}$, it is easy to check that $E_{a}$ is an interval if $1 / 2 \leq a<1$. Consequently, if $1 / 2 \leq a<1$, $E_{a}+x E_{a}$ is an interval for all $x$ in $[0,1]$. The author's goal is to determine the topological structure of $E_{a}+x E_{a}$ for all $a$ in $(0,1 / 2)$ and all $x$ in $[0,1]$. The remainder of this paper will give some partial results toward this end.

Corollary 2. Assume $a \in(0,1 / 2)$ and $a \leq x \leq 1$.

(i) $E_{a}+x E_{a}$ is an interval if and only if $1-2 a \leq x \leq a /(1-2 a)$.

(ii) $E_{a}+x E_{a}$ is homeomorphic to the Cantor set if $a /(1-2 a)<x<$ $1-2 a$.

Proof. This is an immediate consequence of Theorem 3 with $a_{n}=a^{n}$, noting that $r_{n}=a^{n+1} /(1-a)$ and $a_{n}>r_{n}$ for all $n$ since $a \in(0,1 / 2)$.

Lemma 1. Assume $a \in(0,1 / 2)$ and $z \in[0,1]$. If $E_{a}+z E_{a}$ is an interval and $z a \geq 1-2 a$, then $E_{a}+z a E_{a}$ is also an interval.

Proof. It is easy to check that $E_{a}=a E_{a} \cup\left(a+a E_{a}\right)$. Hence $E_{a}+z E_{a}=$ $a\left(E_{a}+z E_{a}\right) \cup\left(a+a\left(E_{a}+z E_{a}\right)\right)$. Now $a\left(E_{a}+z E_{a}\right)$ is an interval with length

$$
a(a+z a) /(1-a) \geq a(a+1-2 a) /(1-a)=a .
$$

Therefore $E_{a}+z a E_{a}$ is also an interval.

Theorem 4. Assume $a \in[1 / 3,1 / 2]$ and $1-2 a \leq x \leq 1$. Then $E_{a}+x E_{a}$ is an interval.

Proof. There exists a positive integer $k$ such that $a^{k+1} \leq x<a^{k}$. Hence $a \leq x / a^{k}<1$. Setting $z=x / a^{k}$, we have $z>x \geq 1-2 a$ and 
$z \leq a /(1-2 a)$ since $a \geq 1 / 3$. Therefore by Corollary $2(\mathrm{i}), E_{a}+z E_{a}$ is an interval. By the lemma, $E_{a}+z a E_{a}$ is an interval. Invoking the lemma $k-1$ times we conclude that $E_{a}+x E_{a}$ is an interval.

TheOREM 5. Assume $a \in(0,1 / 2)$. If $a^{k}(1-2 a) \leq x \leq a^{k+1} /(1-2 a)$ for some positive integer $k$, then $E_{a}+x E_{a}$ is a finite union of intervals.

Proof. Let $z=x / a^{k}$. Then $1-2 a \leq z \leq a /(1-2 a)$ and by Corollary 2(i) and Theorem $4, E_{a}+z E_{a}$ is an interval. Let

$$
F=\left\{\sum_{i=1}^{k} \varepsilon_{i} a^{i}: \varepsilon_{i}=0 \text { or } 1(i=1,2, \ldots, k)\right\} .
$$

It is easy to check that

$$
E_{a}=\bigcup_{f \in F} f+a^{k} E_{a}
$$

Hence

$$
E_{a}+x E_{a}=\bigcup_{f \in F} f+a^{k}\left(E_{a}+z E_{a}\right)
$$

which is a finite union of intervals.

Corollary 3. If $a \in[1 / 3,1 / 2]$, then $E_{a}+x E_{a}$ is a finite union of intervals for all $x \in[0,1]$.

P r o o f. It is easy to check that if $a \in[1 / 3,1 / 2)$, the sequence of intervals

$$
\left[a^{k}(1-2 a), a^{k+1} /(1-2 a)\right] \quad(k=1,2, \ldots)
$$

is overlapping and the union is $(0,1]$. The conclusion then follows from the theorem.

Corollary 2(i), Theorems 4 and 5 and Corollary 3 give all the information presently known about the values of $a \in[0,1 / 2]$ and $x \in[0,1]$ for which $E_{a}+x E_{a}$ is an interval or a finite union of intervals. The information known about when $E_{a}+x E_{a}$ is homeomorphic to the Cantor set is given in Corollary 2(ii) and the following theorem.

TheOrem 6. Assume $a \in(0,1 / 2)$. If $a^{k+1} /(1-2 a)<x<a^{k}(1-2 a)$ for some positive integer $k$, then $E_{a}+x E_{a}$ is homeomorphic to the Cantor set.

Proof. Let $z=x / a^{k}$. Then $a /(1-2 a)<z<1-2 a$ and by Corollary 2(ii), $E_{a}+z E_{a}$ is homeomorphic to the Cantor set. In the notation of the proof of Theorem 5 ,

$$
E_{a}+x E_{a}=\bigcup_{f \in F} f+a^{k}\left(E_{a}+z E_{a}\right)
$$

which is a finite union of sets homeomorphic to the Cantor set and hence is itself homeomorphic to the Cantor set. 
The following theorem gives the only known values of $a \in[0,1 / 2]$ and $x \in[0,1]$ for which $E_{a}+x E_{a}$ is homeomorphic to the set $T$ of Theorem 1 .

ThEOREM 7. $E_{1 / 4}+(1 / 4)^{n}(2 / 3) E_{1 / 4}$ is homeomorphic to the set $T$ of Theorem 1 for $n=0,1,2, \ldots$

Proof. The set $T$ of Theorem 1 is $3 E_{1 / 4}+2 E_{1 / 4}=3\left(E_{1 / 4}+(2 / 3) E_{1 / 4}\right)$, which proves the theorem for $n=0$. By the reasoning in the proofs of Theorems 5 and 6 , the other sets in the theorem are finite unions of contractions of $T$. By reasoning similar to that in Theorem 3, the sets can be viewed as sets of subsums of infinite series. Since the sets contain infinitely many intervals, by Theorem 1 , the sets must be homeomorphic to $T$.

\section{REFERENCES}

[1] E. Barone, Sul codominio di misure e di masse finite, Rend. Mat. Appl. 3 (2) (1983), 229-238.

[2] J. A. Guthrie and J. E. Nymann, The topological structure of the set of subsums of an infinite series, Colloq. Math. 55 (1988), 323-327.

[3] H. Hornich, Über beliebige Teilsummen absolut konvergenter Reihen, Monatsh. Math. Phys. 49 (1941), 316-320.

[4] S. Kakeya, On the partial sums of an infinite series, Tôhoku Sci. Rep. 3 (4) (1914), $159-164$.

[5] S. Koshi and H.-C. Lai, The ranges of set functions, Hokkaido Math. J. 10, special issue (1981), 348-360.

[6] P. K. Menon, On a class of perfect sets, Bull. Amer. Math. Soc. 54 (1948), 706-711.

[7] J. E. Nymann, The sum of the Cantor set with itself, Enseign. Math. 39 (1993), $177-178$.

[8] M. Pavone, The Cantor set and a geometric construction, ibid. 35 (1989), 41-49.

[9] J. Shallit, Q785, Math. Mag. 64 (5) (1991), 351 and 357.

Added in proof (September 1994):

[10] P. Mendes and F. Oliveira, On the topological structure of the arithmetic sum of two Cantor sets, Nonlinearity 7 (1994), 329-343.

This recent paper contains interesting results which are related to the results in the present paper.

DEPARTMENT OF MATHEMATICS

THE UNIVERSITY OF TEXAS AT EL PASO

EL PASO, TEXAS 79968

U.S.A.

Reçu par la Rédaction le 19.1.1994;

en version modifiée le 12.7.1994 